\title{
Radiometric measurements of the parameters of hydrotransport in industrial pipe-lines
}

\author{
by K. Przewlocki and P. Nizegorodcew \\ Institute of Nuclear Techniques \\ Academy of Mining and Metallurgy, Krakow, Poland
}

\section{Introduction}

An increased pipe transportation of solids is the reason of the development of measuring methods of the basic flow parameters such as mean density, flow velocity, slip velocity, spatial distribution of solids and head losses. Apart from the head losses all the other parameters can be measured by means of radiometric methods.

The measurements of density and spatial distribution of concentration are based on the well-known effect of exponential attenuation of radiation beam intensity in matter. An injection of labelled fluid of solids into mixture stream allows an estimation of their mean velocities with accuracy about $1 \%$

\section{Mean density measurements}

The idea of measurement is shown in Figure 1, where a simplified diagram of the measuring system is presented. The radiation source, placed in a safety container emits a well-colimated gamma ray beam penetrating the pipeline perpendicularly to the pipe axis, along its diameter. Owing to absorption in the pipe walls and in the flowing mixture, the radiation intensity decreases while transversing the pipeline according to the exponential absorption law which in given geometrical conditions and in the case of full-rate feeding takes the form:

$$
\mathrm{J}=\mathrm{J}_{0} \exp -\left(\mu_{s} \mathrm{P}_{\mathrm{s}} x_{s}+\mu_{w} \mathrm{P}_{w} x_{w}+2 \mu_{i} \rho_{i} d_{i}\right)
$$

where $\mu_{s}, \mu_{w}$ and $\mu_{i}$ there are mass absorption coefficients of solids, water and iron, $\rho_{s}, \rho_{w}$ and $\rho_{i}$-densities of solids, water and iron respectively, $x_{s}$ and $x_{w}$-equivalent solids and water thicknesses in the stream cross-section, $d_{i}$-thickness of the pipe wall, $\mathbf{J}_{0}$-primary radiation intensity and $\mathbf{J}$-radiation intensity reaching the detector.

Taking into account the condition of full-rate feeding simple relations:

$$
x_{s}+x_{w}=\mathrm{D} \quad \text { and } \quad x_{s} / x_{w}=e
$$

may be formulated, where $\mathrm{D}$ is the pipe diameter and $e$ represents the volumetric ratio of the mixture. An exponent factor related to the absorption in pipe walls is cons. tant. Thus, the above expressions (1) may be rewritten in the form:

$$
\mathbf{J}=\mathrm{J}_{0} \alpha \exp \left(-\mu_{s} \rho_{s} x_{s}-\mu_{w} \rho_{w} x_{w}\right)
$$

where:

$$
\alpha=\exp \left(-2 \mu_{i} \rho_{i} x_{i}\right)
$$

One may also introduce the effective mass absorption coefficient $\mu_{e f f}$ and average density mixture $\rho_{m}$ expressed as follows:

$$
\begin{gathered}
\mu_{e f f}=\frac{\mu_{s} \rho_{s} e+\mu_{w} \rho_{w}}{\rho_{s} e+\rho_{w}} \\
\rho_{m}=\frac{\rho_{s} e+\rho_{w}}{e+1}
\end{gathered}
$$

In conditions under consideration it allows the description of the absorption law in a clear form:

$$
\mathrm{J}=\alpha \mathrm{J}_{0} \exp -\left(\mu_{\ell j} P_{m} \mathrm{D}\right)
$$


One must, however, remember that in general the average density $p_{m}$ as well as the effective absorption coefficient $\mu_{c f f}$ are functions of volumetric ratio $e$.

Measurement of the mixture density results in average frequency of pulses generated in the radiation detector. This form of obtaining information makes a digital-analogue converter indispensable. This converter is usually in the form of a pulse integrator. As the result of this conversion what is obtained at the output is the voltage or current representation given by the relation:

$$
\mathrm{U}_{o u t}=\mathrm{K} \alpha \mathrm{J}_{0} \exp -\left(\mu_{e j p} p_{m} \mathrm{D}\right)
$$

in which $\mathrm{K}$ denotes the coefficient of transformation and amplification of the signal.

The statistical and apparatus errors define precisely and sufficiently the accuracy of measuring the density of uniform fluids. The problem, however, becomes more complicated when a nonuniform two-phase liquid density is measured, the components of which flow in the pipeline with different average velocities. This is the reason why one must distinguish between the density registrated by the measuring gauge installed at the pipeline, communly called "in-line" density and the density found at the output of the installation, called "delivered" density.

This phenomenon of the solid particles slip related to the transporting liquid is the result of the interaction of moving liquid with the passive solid particles, interaction of solid particles and their interaction with the wall layer.

The difference between the "in-line" and "delivered" densities is not very significant. Korbel has shown that in the most unfavorable conditions in sand transport for mining back fill the discrepancy between their values does not exceed 3\% [1]. Commonly, the industrial density meters have less than $3 \%$ error, so that in mean transport density measurements $3 \%$ may be considered as the accuracy for radiometric density gauges of the sand-water mixtures. Where slip velocity is negligible, statistical, apparatus and calibration errors are dominating.

Figure 2 shows the example of the record of the mean density obtained from the density meter for the stowing mixture control made at the Institute of Nuclear Techniques.

\section{Spatial distribution of solids concentration}

The research carried on the spatial distribution of solids is one of the most important issues of the unhomogeneous fiuids flow in pipes. Stationary distribution of solids occurs in the pipe cross-section provided the density of hydromixture at the inlet is constant in time. Density patterns may be thus measured by means of scanning of the pipe cross-section. At the Institute of Nuclear Techniques a special apparatus was constructed for this purpose.

Its scanning device performs a rotary motion around the pipe and simultaneously approaches its axis [2]. The idea of the measuring head is shown in Figure 3. As a result of the measurement a curve is obtained. It represents a gamma ray absorption along an infinite number of chords



1/ A schematic diagram of density measurement.

of a given pipe cross-section, tangential to the Archimedian spiral. The spiral is described by the equation:

$$
p=h / 2 . \Phi
$$

In the described apparatus the duration of the total run measurement is about $3 \mathrm{mn}$, the width of the gamma beam and spiral thread are $5 \mathrm{~mm}$. To determine the density pattern of the hydromixture flow a set of chords transversing the pipe at certain angles must be chosen. A gamma ray absorption on an empty pipe must be also performed earlier as a calibration measurement. The gamma radiation originates from the Cs-137 photopeak.

The relative intensity of gamma radiation passing the pipe cross-section along a chosen chord can be described by the formula:

$$
\delta(L)=\int_{\dot{L}} \mu(L) d l
$$

where $\delta$ is the logarithm of the attenuation of gamma radiation along the chord:

$$
\delta=\ln \mathrm{J}_{0} / \mathrm{J}
$$

and $\mu$ is a linear absorption coefficient, which is a function of density. It can also depend on geometrical variable L, and perhaps on grain size and grain shape of solids [3].

Each chord described by the parameter $\mathrm{L}$ can be also described by two variables $x, y$, coordinates in the crosssection plane. Hence, linear absorption coefficient depends on the same two variables:

$$
\mu(\mathrm{L})=\mu(x, y)
$$

A set of experimental data has the form of a set of mean chord absorption coefficients $\mu_{k}$ or mean chord densities $\rho_{k}$, when $k$ denotes a number of chord. It enables to solve an integral equation (9) i.e. to find an absorption coefficient distribution in the cross-section under consideration $\mu(x, y)$. This distribution can be approximated by a polynomial of the form:

$$
\mu(x, y)=\sum_{i, j=0}^{n} a_{i j} x^{n-i} y^{, i}
$$



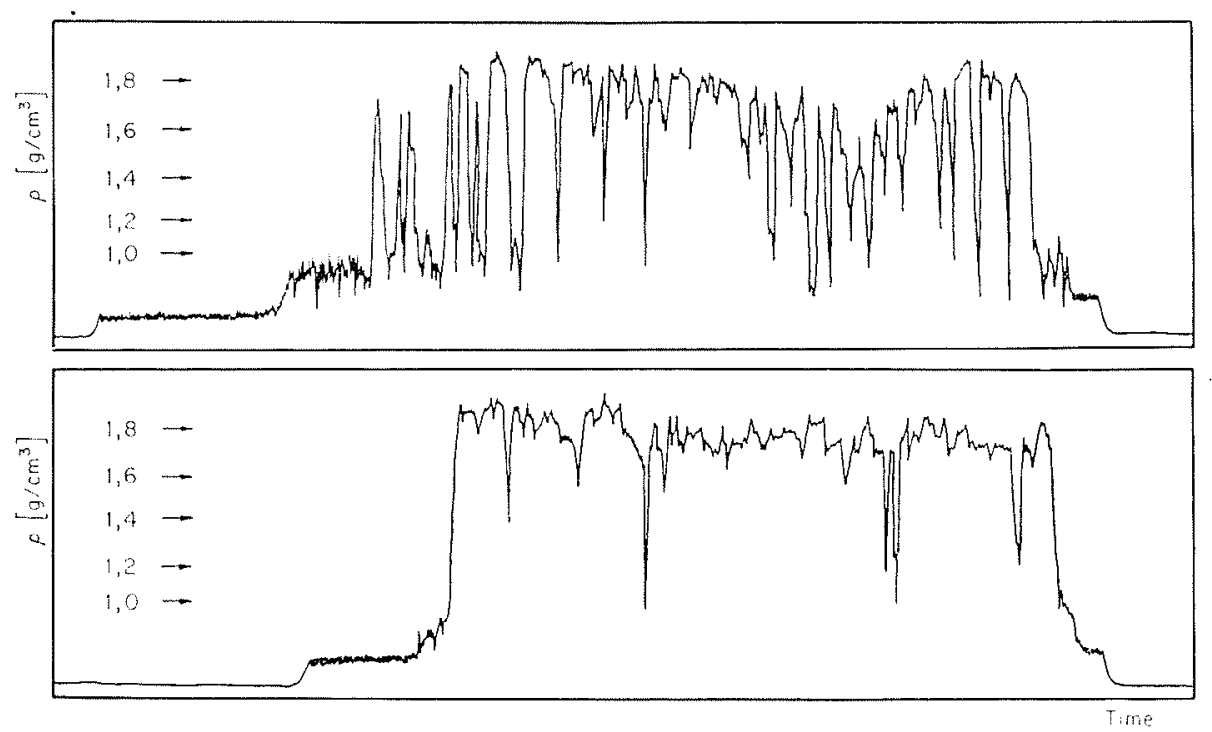

2/ Example of the mean density record.

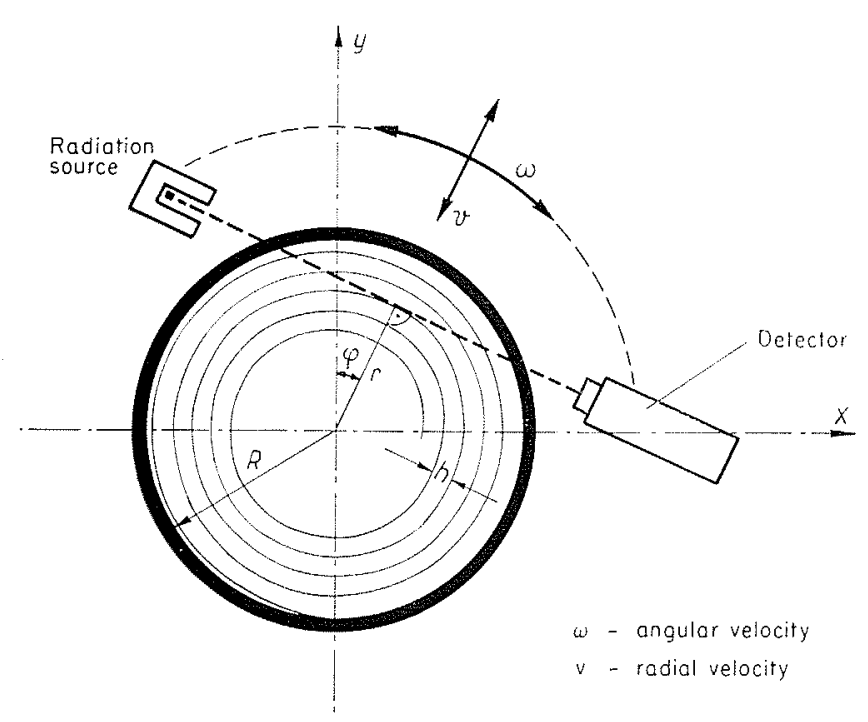

3/ Scanning system for a spatial density distribution.

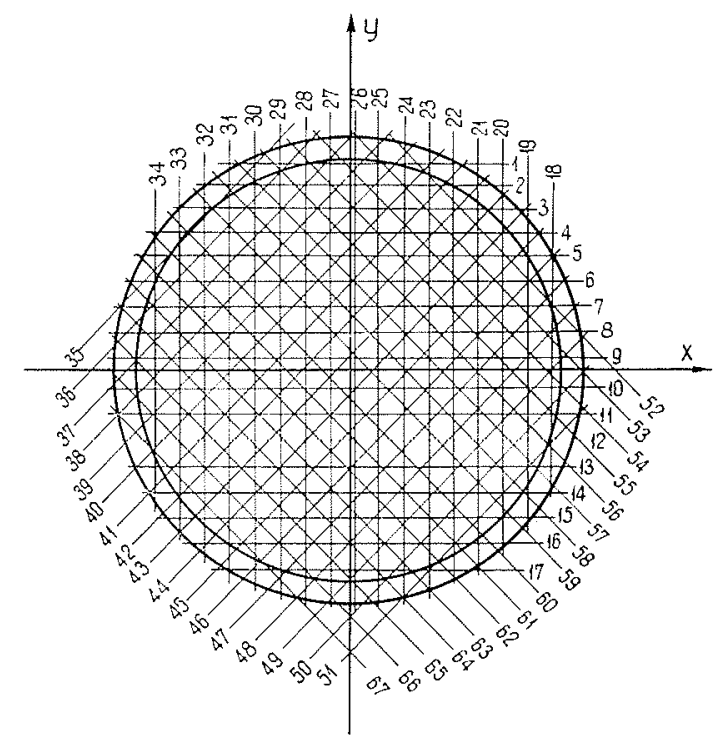

4/ Chord net for an evaluation of the spatial density distribution. 
Inserting the equations of the chosen chords:

$$
y_{k}=\alpha_{k} x+\beta_{k}
$$

$\mu(L)$ becomes a function of one variable $x$. Of course, this $\mu(\mathrm{L})$ distribution is related to the distribution of density $P(L)$ and above evaluation can be also executed with the density data. The obtained system of equations unequivocally determines the coefficients $a_{i j}$ of the polynomial. It has been assumed that the optimum approximation is achieved by the use of a fourth-order polynomial.

It should be pointed that the accuracy of approximation i.e. error of final results depends also on the choice of number and arrangement of chords. On the other hand, the final error depends on the inaccuracy of attenuation measurements. As it was mentioned above it is necessary to take into account that the $\mu\left(\mathrm{I}_{-}\right)$and $p(\mathrm{~L})$ correspondence depends on geometrical conditions, especially in the region close to the pipe wall.

The set of equations is solved with the aid of a digital computer. After the calculation of the subsequent polynomial coefficients, a well fitted value of density is attached to each point of the cross-section of the measured pipe. Finally, a complete density or concentration pattern at the pipe cross-section is obtained.

To interpret the results of measurements obtained in stowing pipes of diameter $185 \mathrm{~mm}$ a set of 67 equations is used. The chosen chords form a net of squares about $10 \times 10 \mathrm{~mm}^{2}$. This net of chords is shown in Figure 4 . To simplify the evaluation three types of chords were taking into account: vertical, horizontal and inclined at $45^{\circ}$. The values of the mean density $\rho_{i}$ are calculated from the scanning head records.

Each chord is identified on the record tape with the accuracy of $2^{\circ}-4^{\circ}$, and corresponding mean density $p_{l}$ are calculated. Scanning measurements of simple models of density distribution have shown the effect of the dynamic retarding shift of the recorded value versus the geometrical position of the chord. Such an effect can be explained by the influence of the time constant of a registrating integrator.

The gamma ray beam diameter significantly infuences the total error. It is particularly unfavorable in the region close to pipe walls. It is obvious that total error is relatively small in central part of the cross-section, and increases in wall region. Uniformity of chords net as well as the method of polynomial approximation, its order and the fitting procedure are responsible for other errors.

The total error evaluated from the model measurements ranges from $1 \%$ in the centre of the cross-section to about $5 \%$ in the wall region. Figure 5 shows an example of the spatial distribution of density, obtained from the stowing pipeline measurements.

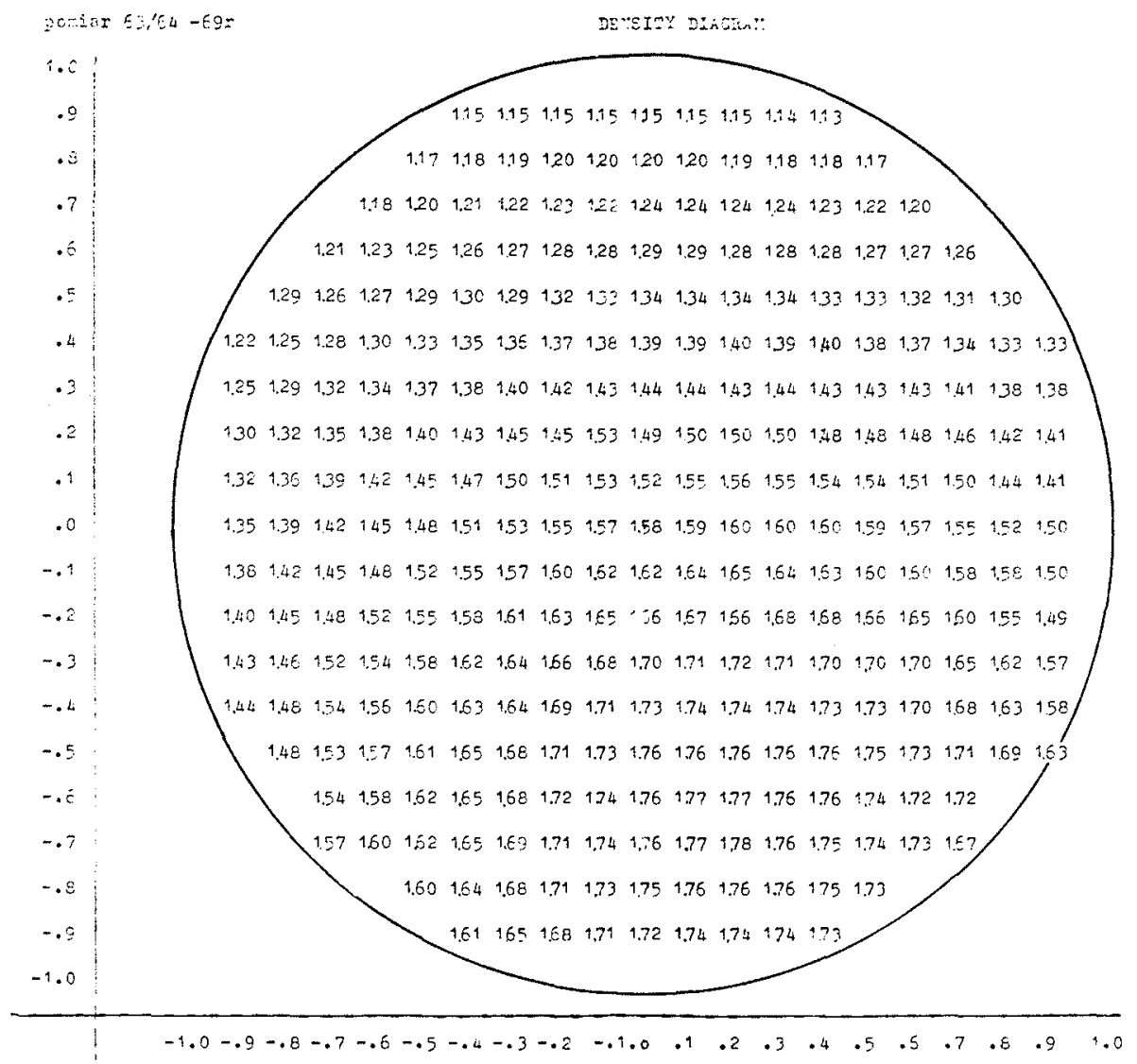

5/ Example of a density diagram in pipe cross-section. 


\section{Flow velocity measurements}

The method employing the time of peak passing was found to be the most suitable for the evaluation of hydromixtures flow velocity [4]. The following methods to accomplish labelling of solids have been used: irradiation of the actual process material to induce useful activities, affixing a radioactive substance to the surface of the process material by adsorption or impregnation, mechanical tagging with a radioactive source, making a physical imitation of the material under study which is easily rendered radioactive. The first two methods are generally used when a large portion of a fine-grain material is to be labelled for obtaining statistically valid results, whereas the third and the fourth alternative are used when physical similarity is easy to realize.

The labelled material is introduced into the pipeline by means of special injectors. Different types of injectors have been developed both for liquid and solids [5]. Two gamma ray detectors are placed at the distance greater than at the "mixing distance" along the pipeline. The so called "mixing distance" is the distance between the injector and first detector, ensuring the homogeneous distribution of the tracer over the whole pipe cross-section at the required level of accuracy. After injection of a radioactive tracer two peaks are recorded by both paralelly connected detectors.

The mean flow velocity can be determined as:

$$
\left(x_{2}-x_{1}\right) /\left(t_{e q 2}-t_{e q 1}\right)
$$

where $x_{2}$ and $x_{1}$ are the coordinates of the detector positions along the pipe and $t_{e q_{2}}$ and $t_{e q 1}$ are the equivalent transit times of the second and first peak respectively. According to the most frequently used methods the equivalent transit time $t_{e g}$ is defined as the time coordinate of the centre of gravity of the activity peak [6] or as the time coordinate of the maximum point of the activity peak or of the $1 / 3$ of the peak width [7 and 8]. The choice of the proper and most convenient method of the estimation of transit time has to be made with respect to the actual shape of the activity peaks. The first method was chosen for the most diffused and stretched peaks whereas the second may be applied for the sharper and more symmetrical peaks.

The radiometric method of the flow velocity determination permits to perform absolute measurements of the flow velocity with relatively high accuracy in comparison with other applicable methods. The total activity of labelled liquids and solids is very little, hence contamination of plant installations and technological wastes are negligible.

Specially processed glass is most suitable for simulation of sand grains in either industrial and laboratory experiments [9]. Salts of sodium, such as $\mathrm{Na}_{2} \mathrm{CO}_{3}$ marked by $\mathrm{Na}-24$ are used to label liquids.

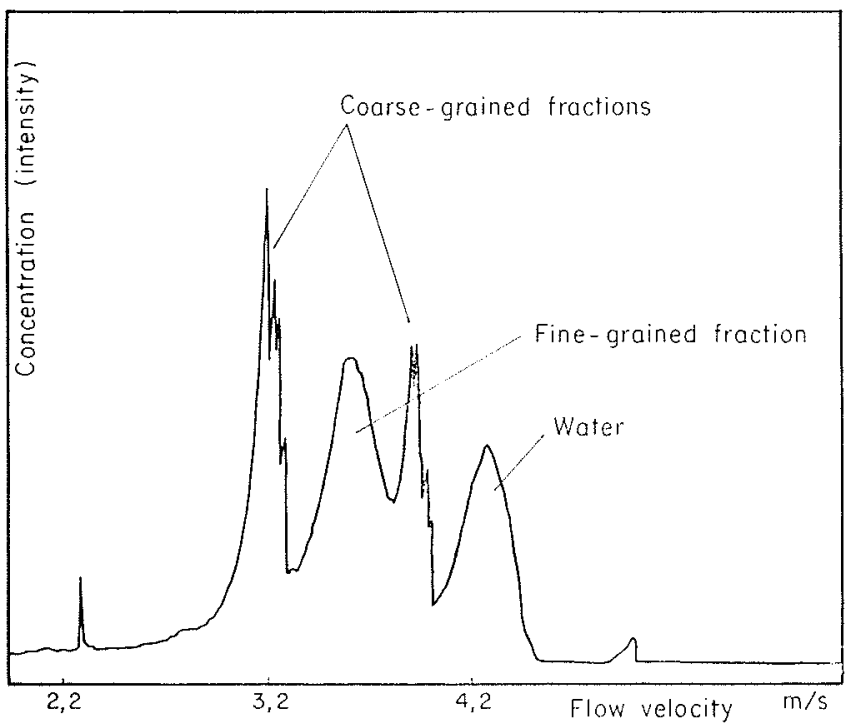

6/ Distinct peaks related to several phase velocilies of solids in pipeline transport.

A wide range of activation possibilities enables to obtain data about various fractions of flowing media as well as of slip velocities and other transport phenomena. E.g. the distinct velocities of few solid fractions of stowing hydromixture are evidently apparent in Figure 6 which represents an example of record obtained by means of method described above.

The accuracy of the method is high, about $1 \%$. It is due mainly to the error of the measurements of the distance between two peaks and its coordinates.

\section{Further remarks}

All presented methods are applicable in various industrial and research investigations [10]. Main characteristics of various types of flow can be estimated in complex experiments which make use of all presented methods. More advanced experiments e.g. analysis of the shape of time distribution of passing peak concentration enable the research on dynamics of transport processes in various industrial plants as well as in laboratories.

The methods described above having a relatively high accuracy may be also used for the standardisation of the classical methods. 


\section{Bibliography}

[1] KORBEL (K.). - The applicability of radiogauges for measuring the density of flowing solid-liquid mixtures. Reports of the Institute of Nuclear Physics, Cracow, $\mathrm{n}^{\circ} 704 / \mathrm{PH}(1970)$.

[2] Michalik (A.), Praxmayer (T.), Przewlocki (K). - «A radiometric method of determination of the spatial distribution of density in closed media. Nukleonika, 13, 1 (1968), 107.

[3] Gyurcsak (J.), UMrastowski (K). - The absorption of gamma radiation in inhomogeneous media. INT Report, Cracow (in print).

[4] Przewlocki (K), Michalik (A.), Nizegorodcew (P.), Korbel (K). - Investigation of the flow of inhomogeneous fluids in pipe-lines by means of radioisotopes. Report on Research Agreement, 465/CF, I.A.E.A. (1970).
[5] Dobrzanski (J.), Korbel (K), Owsiak (T.). - Atommaja Energia, 30, 4 (1971), 393 (in russian).

[6] Brshoff (K. B.), Levenspiel (O.). - Chem. Eng. Sci., 17 (1962), 245.

[7] Clayton (C. G.). - Radioisotope tracers in industry and geophysics. Symp. Proc. I.A.E.A., Vienna (1967), 563.

[8] Taylor (G. 1.). - Proc. Roy. Soc., A223 (1954), 446.

[9] Korbel (K), Przewlock (K), Michalik (A.), Nizegorodcew (P). - Determination of some kinematic flow characteristics.. INP. Reports, Cracow, $\mathrm{n}^{\circ} 717 / \mathrm{PL}(1970)$.

[10] LJUNGGREN (K.). - Radioisotope tracers in industry and geophysics. Proceedings of the I.A.E.A. Symp., Prague (1966), 303. 